\title{
Evaluation of serum and urine neutrophil gelatinase-associated lipocalin and cystatin C as biomarkers of acute kidney injury in horses
}

\author{
Natalia Siwińska ${ }^{1 凶},{\text { Agnieszka } \dot{Z Z k}^{2} \text {, Urszula Pasławska }}^{1,3}$ \\ ${ }^{1}$ Department of Internal Medicine and Clinic of Diseases of Horses, Dogs and Cats, Faculty of Veterinary Medicine, \\ Wroclaw University of Environmental and Life Sciences, 50-375 Wrocław, Poland \\ ${ }^{2}$ Department of Immunology, Pathophysiology and Veterinary Preventive Medicine, \\ Wroclaw University of Environmental and Life Sciences, 50-375 Wrocław, Poland \\ ${ }^{3}$ Veterinary Institute, Faculty of Biological and Veterinary Sciences, \\ Nicolaus Copernicus University in Torun, 87-100 Toruń, Poland \\ natalia.siwinska@upwr.edu.pl
}

Received: November 19, $2020 \quad$ Accepted: April 22, 2021

\begin{abstract}
Introduction: Diagnosis of acute kidney injury (AKI) in horses is difficult at the subclinical stage, due to nonspecific clinical signs. The aim of this study was to evaluate the concentrations of selected serum and urinary biomarkers in healthy horses, horses at risk of AKI, and those with clinical AKI. Material and Methods: Thirty healthy horses, 30 horses at risk of AKI and 11 horses with clinical AKI and azotaemia were included in the study. Serum and urinary neutrophil gelatinase-associated lipocalin (NGAL) and cystatin $\mathrm{C}$ were measured using commercially available enzyme immunoassay tests. Results: The median and (in parentheses) first and third quartile concentrations of selected biomarkers in healthy horses, horses at risk of AKI and horses with AKI were respectively as follows: serum cystatin $\mathrm{C}-0.25(0.19-0.37), 0.23(0.15-0.37)$ and $0.61(0.37-1.13) \mathrm{mg} / \mathrm{L}$; serum NGAL - 50.5 (38.8-58.8), 51.1 (40.4-66.9) and 98.1 (59.4-128.2) ng/mL; urinary NGAL - 20.7 (17.9-24.5), 32.3 (32.7-55.8) and 36.6 (26.8$89.9) \mathrm{ng} / \mathrm{mL}$; and urinary cystatin $\mathrm{C}-0.1(0.07-0.13), 0.13(0.1-0.2)$ and $0.34(0.22-0.37) \mathrm{mg} / \mathrm{L}$. There were significant differences in the concentration of all biomarkers between the healthy and AKI-affected horses. Conclusion: Horses with AKI all had biomarker concentrations higher than the healthy horses. None of the biomarkers made azotaemia recognisable in all affected horses. The obtained results indicate the need to create a serum and urinary biomarker panel to detect AKI.
\end{abstract}

Keywords: acute kidney injury, cystatin C, nephrotoxicity, NGAL, NSAIDs.

\section{Introduction}

Acute kidney injury (AKI) is a syndrome of sudden organ damage, often secondary to other primary diseases. The early stage of injury itself is not always associated with obvious organ dysfunction, which makes diagnosis challenging. Unfortunately, even a mild, subclinical kidney injury can lead to severe consequences, such as acute kidney failure or chronic kidney disease (12). This condition, underestimated in horses in the past, is quite common, especially in patients undergoing intensive care $(6,19)$. The most common causes of AKI in horses are haemodynamic changes (dehydration or hypovolaemia), endotoxaemia or systemic inflammatory response syndrome, and the use of potentially nephrotoxic drugs (especially aminoglycosides and nonsteroidal anti-inflammatory drugs - NSAIDs) $(1,11,25)$. Since the early stages of AKI do not give specific clinical signs and manifest only in exacerbation of the primary disease, non-specific urinary abnormalities and slight to no change in ultrasound, there is a need to identify sensitive and specific biomarkers of this condition (22). Currently, the diagnosis is mainly based on detection of an increase in the serum creatinine concentration, which occurs relatively late in the AKI process and is dependent on many non-renal factors. In human medicine, many serum and urinary biomarkers are used to detect AKI. These include dysfunction and injury biomarkers such as cystatin $\mathrm{C}$ and neutrophil gelatinase-associated lipocalin (NGAL) (30). In equine medicine such biomarkers are not in widespread use, and in the 
literature there are only limited studies which investigated cystatin $\mathrm{C}$ and $\mathrm{NGAL}$ for evaluation of renal function in horses $(1,9,27)$.

The main goal of this study was to report and compare the concentrations of serum and urinary cystatin $\mathrm{C}$ and NGAL in healthy horses, horses at risk of AKI and horses with clinical AKI. The secondary goal was to assess the relationship between these and more conventional renal dysfunction biomarkers such as serum creatinine and urea, urinary protein, the gamma-glutamyl transpeptidase (GGT) to creatinine ratio, and fractional excretion of sodium (FENa). Additionally, this research sought to establish which biomarkers could be used to detect AKI and examine how potentially nephrotoxic factors such as gastrointestinal diseases and drugs affect the kidneys.

\section{Material and Methods}

For this investigation, samples from a previously published study were used. A detailed description of the study design, group constitution and additional results was provided by Siwińska et al. (23).

Animals: The study was conducted on 71 adult warmblood horses. The animals were divided into three groups: group I, the non-AKI group; group II, the AKIrisk group; and group III, the AKI group. The history of all the horses was learned from the owners, and each animal's body weight was measured using a horse measurement tape or scale. All horses underwent a full clinical examination, urinary tract ultrasound examination, and urine and blood analysis. The non-AKI group consisted of 30 healthy horses (15 mares and 15 geldings) between 3 and 29 years old (mean 14.7, SD 8.4). The inclusion criteria were good body condition, no local or general disease at the time of examination or in an at least 6-month period before and after the research, no drug administration in the 6-month period before the study, bloodwork and urinalysis results within the laboratory reference ranges and no urinary tract lesions visible in transabdominal ultrasonography. The AKI-risk group also contained 30 horses (15 mares and 15 geldings) aged 2-27 years (mean 14.7, SD 7.6). This group consisted of three subgroups. Subgroup I was ten horses being treated for colic or gastrointestinal diseases. Subgroup II incorporated ten horses receiving phenylbutazone at a dose of $2.2 \mathrm{mg} / \mathrm{kg}$ body weight (b.w.) every $12 \mathrm{~h}$ orally for 10 days, and subgroup III held ten horses receiving gentamicin at a dose of $6.6 \mathrm{mg} / \mathrm{kg}$ b.w. intravenously for 5 days. Phenylbutazone and gentamicin were administered for a variety of conditions. The inclusion criteria were serum creatinine and urea concentration within the physiological ranges at study commencement, and no systemic manifestation of primary disease such as endotoxaemia, sepsis or systemic inflammatory response syndrome throughout the period of investigation. Serum creatinine concentration could be slightly elevated at the end of the research without this leading to exclusion, but only by no more than $25 \%$ from the baseline. The AKI group assembled 11 horses with clinical AKI (6 mares and 5 geldings) no younger than 2 or older than 20 years (mean 9.4, SD 5.8). Horses in this group were classified as suffering from AKI at the time and had the condition from different primary causes including cardiovascular compromise, administration of potentially nephrotoxic drugs, endotoxaemia, and postanaesthetic rhabdomyolysis. The inclusion criterion was primary acute disease accompanied by azotaemia. An additional criterion was kidney dysfunction confirmed by changes in urinalysis and/or in renal ultrasound parameters. The exclusion criteria were a history of urinary tract dysfunction or disease, congenital anomalies, post-renal azotaemia, and elevated serum creatinine and urea without other signs of primary disease.

Sample collection: Blood was collected from the jugular vein and put into ethylenediaminetetraacetic acid (EDTA) tubes (Medan, Poland) and tubes with a clot activator (Medan, Poland). Urine was collected into a sterile container during spontaneous urination or via aseptic catheterisation of oliguric horses and horses which had undergone laparotomy. Blood and urine for biomarker evaluation were sampled only once from each horse. In AKI-risk colic horses, samples were collected prior to treatment, and in the AKI-risk horses receiving gentamicin or NSAIDs they were collected once treatment was terminated. In the AKI horses, samples were collected at the commencement of treatment.

Laboratory analysis: Haematological examination was performed from an EDTA blood sample using a Scil Vet ABC animal blood counter (Horiba, USA). The serum was divided and placed in $1 \mathrm{~mL}$ Eppendorf tubes (Eppendorf, Germany) and immediately transported to an external veterinary laboratory for biochemical analysis. The serum creatinine and urea concentrations were assessed using an AU680 chemistry analyser (Beckman Coulter, USA) and dedicated reagents. The remaining serum was stored at $-80^{\circ} \mathrm{C}$ until further NGAL and cystatin $\mathrm{C}$ analysis. Fresh urine was analysed for colour, transparency, specific gravity, $\mathrm{pH}$, glucose, blood, acetone, bilirubin and urobilinogen using urine strips (ComboStik, South Korea). The biochemical analysis of urine protein, creatinine, GGT and sodium was performed using the AU680 chemistry analyser and reagents. The urine protein to creatinine ratio and urine GGT to creatinine ratio were calculated, as was FENa using the formula FENa $(\%)=100 \times$ (urine $\mathrm{Na} \times$ serum creatinine/serum $\mathrm{Na} \times$ urine creatinine). Urine samples in $2 \mathrm{~mL}$ volumes were placed in Eppendorf tubes and stored at $-80^{\circ} \mathrm{C}$ until further NGAL and cystatin $\mathrm{C}$ analysis.

Evaluation of NGAL and cystatin $\mathrm{C}$ from frozen serum and urine samples was undertaken in the Department of Biochemistry and Molecular Biology, Faculty of Veterinary Medicine of the Wroclaw University of Environmental and Life Sciences, Poland. Following thawing, the samples were mixed thoroughly 
and centrifuged briefly. Undiluted serum and urine were used to measure NGAL $(\mathrm{ng} / \mathrm{mL})$ and cystatin $\mathrm{C}(\mathrm{mg} / \mathrm{L})$ levels with commercially available ELISA kits (MyBioSource, USA, catalogue numbers MBS087573 and MBS022947), according to the manufacturer's instructions. These kits were developed and validated by the manufacturer for use with serum and urine samples of equine origin. The plates were washed with an ELx50 Automatic Plate Washer (BioTek Instruments, USA), and the absorbance was assessed with a Multiskan EX photometer (Thermo Scientific, USA). For each measurement, the $620 \mathrm{~nm}$ background absorbance was subtracted from the $450 \mathrm{~nm}$ absorbance, and a fourparameter curve fitting was employed to calculate the analyte concentration. All measurements were performed in duplicate.

Statistical analysis. The normality of distribution of the data was analysed using the Shapiro-Wilk test. One-way ANOVA with Fisher's least significant difference post-hoc test was used to compare normally distributed variables. These were presented as average $\pm \mathrm{SD}$, while abnormally distributed variables were presented as median and first and third quartiles. Any differences between groups in quantitative variables were evaluated using the Kruskal-Wallis test with the post-hoc Conover pairwise comparison and the Benjamini-Hochberg procedure. The correlation between variables was assessed using Spearman's correlation coefficient. A receiver operating characteristic (ROC) analysis was performed to assess the value of the sensitivity and specificity of biomarkers to detect AKI. The tests were considered statistically significant if $\mathrm{P}<0.05$. All analyses were performed using $\mathrm{R}$ for Windows software (version 3.6.1) (18).

\section{Results}

The results concerning basic blood and urinary parameters were previously published by Siwińska et al. (23).
The results of the serum and urinary NGAL and cystatin $\mathrm{C}$ measured in the non-AKI, AKI-risk and AKI horses are presented in Tables and Figs 1 and 2. Table 1 also shows the number of horses with biomarker concentrations above the cut-off value. Non-AKI horses and AKI-risk horses receiving potentially nephrotoxic drugs had significantly lower serum and urinary cystatin $\mathrm{C}$ and serum NGAL concentrations than AKI horses. This difference was not observed in the group of AKI-risk horses with colic. Urinary cystatin $\mathrm{C}$ and NGAL were significantly higher in AKI-risk horses with colic and AKI horses compared to non-AKI horses.

The correlations between NGAL/cystatin $\mathrm{C}$ and conventional serum/urine biomarkers of renal dysfunction are presented in Table 3. Only in the case of urinary cystatin $\mathrm{C}$ was there a low positive correlation with serum creatinine. A low correlation with serum urea was demonstrated by serum NGAL and serum and urinary cystatin $\mathrm{C}$. Urinary cystatin $\mathrm{C}$ and NGAL also showed a low positive correlation with urine protein. All tested biomarkers presented low positive correlations with the GGT to creatinine ratio, except for serum cystatin C. Urinary NGAL was the only biomarker which revealed a low positive correlation with FENa.

Table 4 and Figs 3 and 4 contain the results of the ROC analysis of the concentration of NGAL and cystatin $\mathrm{C}$ to detect acute kidney dysfunction in the examined horses.

The best cut-off values for the evaluated biomarkers were 95.2 for serum NGAL, 33.1 for urinary NGAL, 0.53 for serum cystatin C, and 0.2 for urinary cystatin $\mathrm{C}$. The highest sensitivity for the detection of AKI was demonstrated by urinary cystatin $\mathrm{C}$ and the highest specificity was shown by serum NGAL. All biomarkers proved more effective in detecting negative rather than positive results. The best ability to distinguish between normal and abnormal results was demonstrated by urinary cystatin $\mathrm{C}$, whereas in the case of serum NGAL this ability was fair, and for serum cystatin $\mathrm{C}$ and urine NGAL it was moderate.

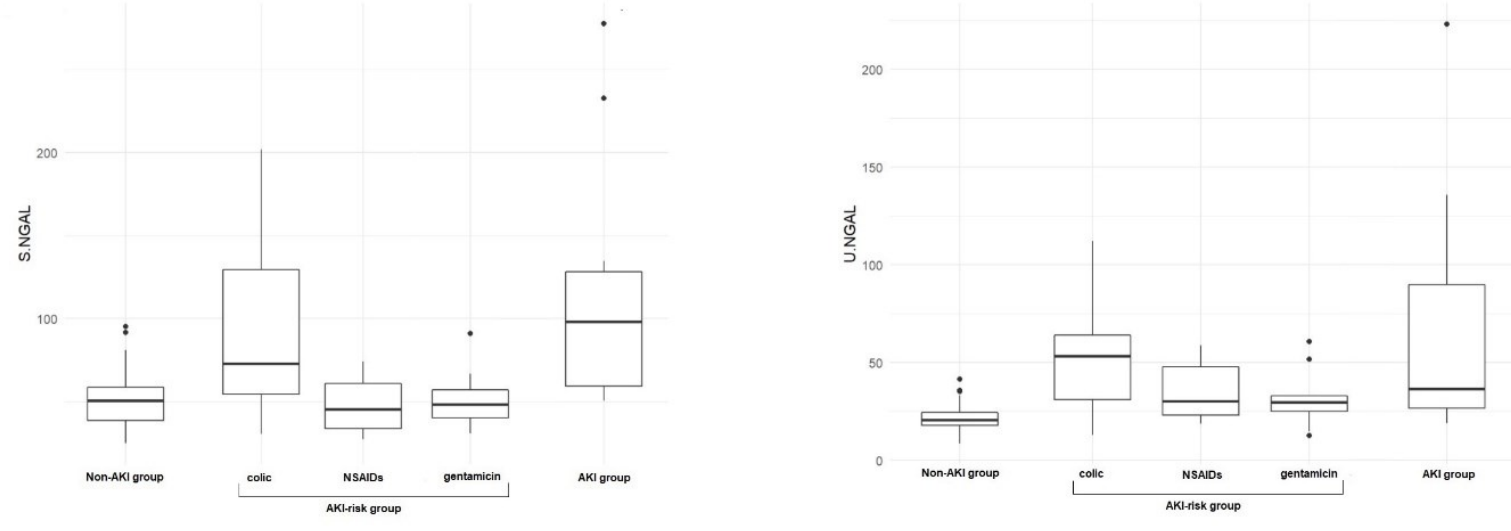

Fig. 1. Results of serum (S) and urinary (U) neutrophil gelatinase-associated lipocalin (NGAL) in healthy horses (non-acute kidney injury (AKI) group), horses at risk of acute kidney injury (AKI-risk group; divided into colic sufferers, recipients of nonsteroidal anti-inflammatory drugs (NSAIDs) and recipients of gentamicin) and horses diagnosed with acute kidney injury (AKI group) 

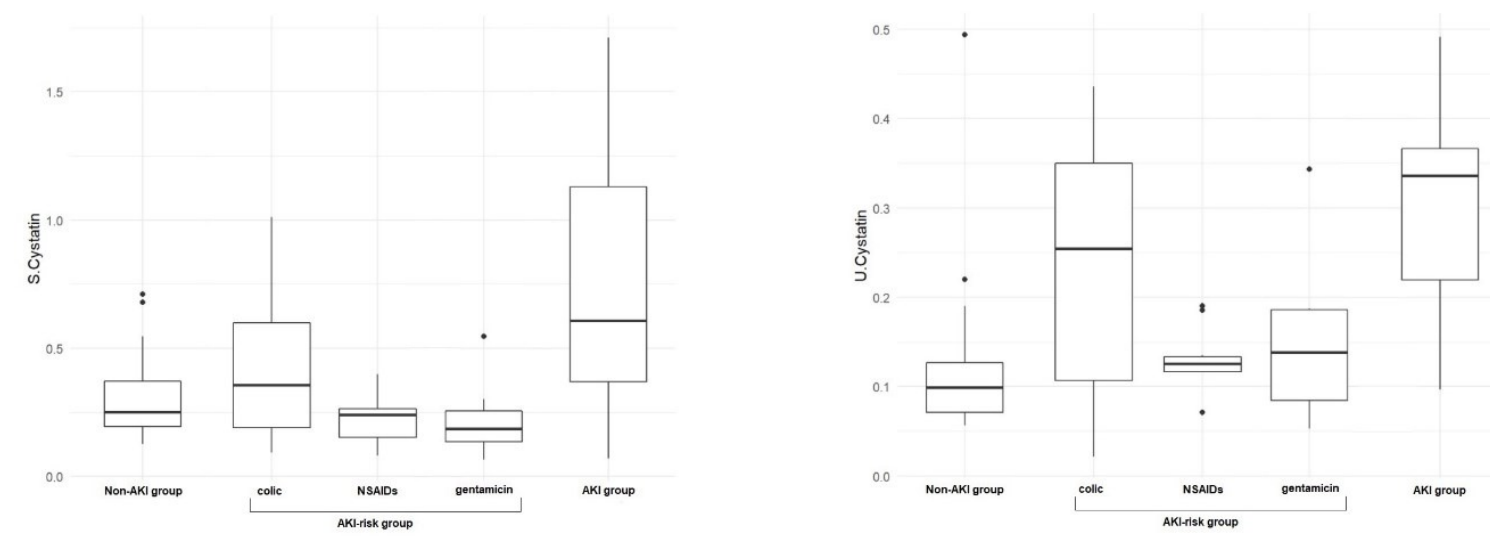

Fig. 2. Results of serum (S) and urinary (U) cystatin $\mathrm{C}$ in healthy horses (non-acute kidney injury (AKI) group), horses at risk of acute kidney injury (AKI-risk group; divided into colic sufferers, recipients of nonsteroidal anti-inflammatory drugs (NSAIDs) and recipients of gentamicin) and horses diagnosed with acute kidney injury (AKI group)

A

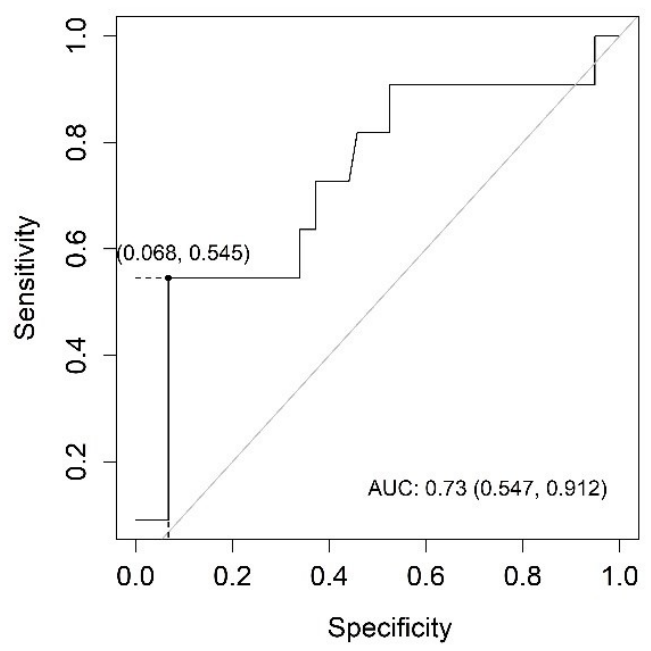

B

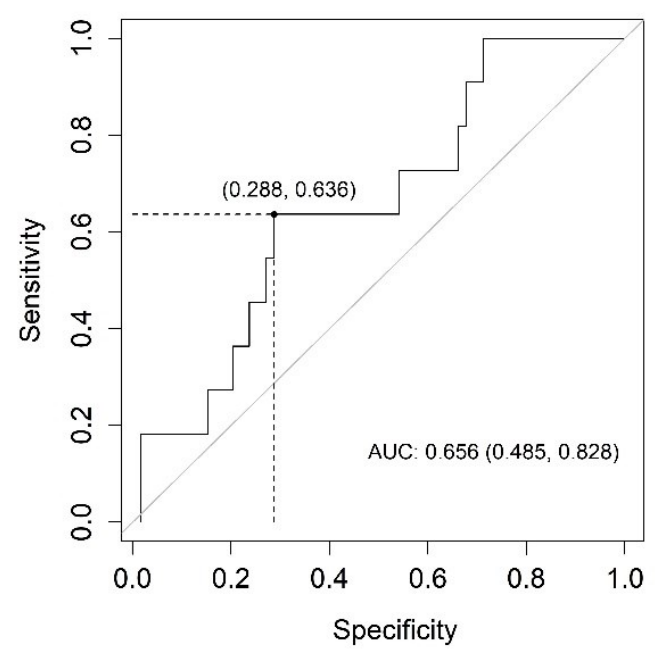

Fig. 3. Receiver operating characteristic (ROC) analysis curve showing the sensitivity and specificity of (A) serum and (B) urinary neutrophil gelatinase-associated lipocalin (NGAL) in relation to the detection of acute kidney dysfunction in the examined horses $\mathrm{AUC}$ - area under ROC

A

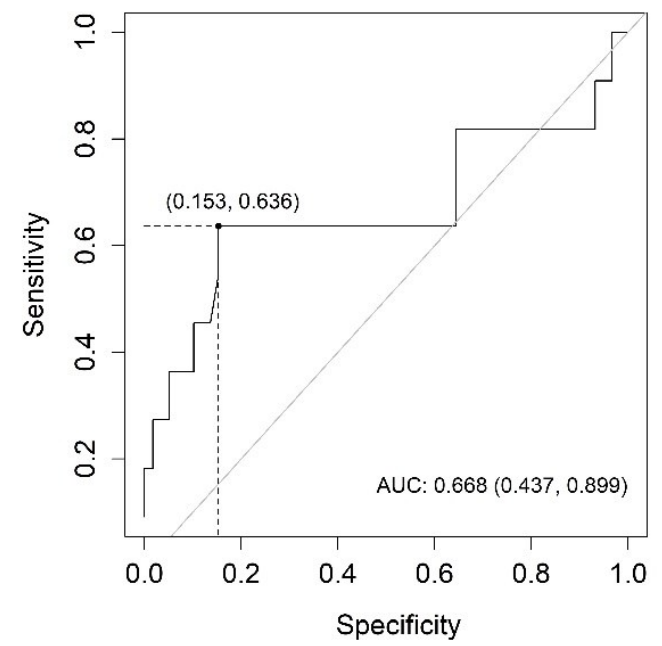

B

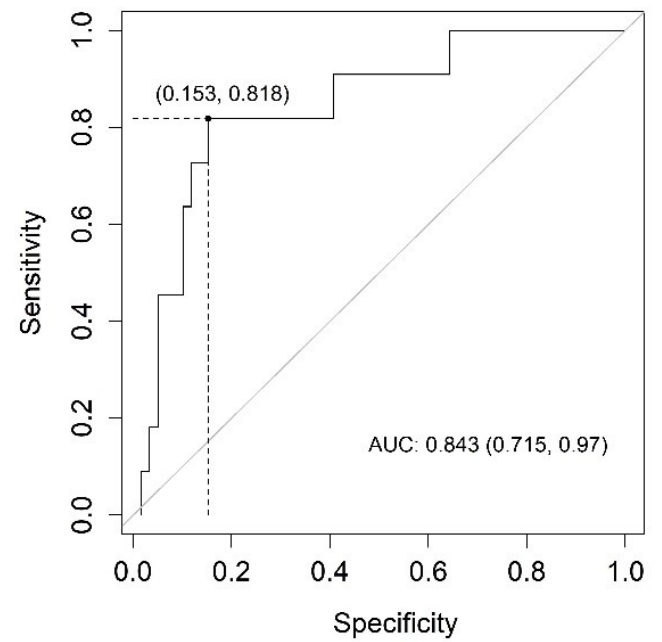

Fig. 4. Receiver operating characteristic (ROC) analysis curve showing the sensitivity and specificity of (A) serum and (B) cystatin C in relation to the detection of acute kidney dysfunction in the examined horses AUC - area under ROC 
Table 1. Concentration of serum and urinary cystatin $\mathrm{C}$ and neutrophil gelatinase-associated lipocalin (NGAL) measured in healthy horses (nonacute kidney injury (AKI) group), horses at risk of acute kidney injury (AKI-risk group; divided into colic sufferers, recipients of nonsteroidal antiinflammatory drugs (NSAIDs) and recipients of gentamicin) and horses diagnosed with acute kidney injury (AKI group)

\begin{tabular}{|c|c|c|c|c|c|c|c|c|c|c|c|c|c|c|c|c|c|c|c|}
\hline \multirow{3}{*}{\multicolumn{2}{|c|}{ Parameter }} & \multirow{2}{*}{\multicolumn{3}{|c|}{ Non-AKI group $(\mathrm{n}=30)$}} & \multicolumn{12}{|c|}{ AKI-risk group $(\mathrm{n}=30)$} & \multirow{2}{*}{\multicolumn{3}{|c|}{ AKI group $(\mathrm{n}=11)$}} \\
\hline & & & & & \multicolumn{3}{|c|}{ Colic $(\mathrm{n}=10)$} & \multicolumn{3}{|c|}{ NSAIDS $(\mathrm{n}=10)$} & \multicolumn{3}{|c|}{ Gentamicin $(\mathrm{n}=10)$} & \multicolumn{3}{|c|}{ All } & & & \\
\hline & & Min-max & $\begin{array}{c}\text { Me (Q1- } \\
\text { Q3) }\end{array}$ & n' & Min-max & $\begin{array}{c}\mathrm{Me}(\mathrm{Q} 1- \\
\mathrm{Q} 3)\end{array}$ & n' & $\operatorname{Min}-\max$ & $\begin{array}{c}\mathrm{Me}(\mathrm{Q} 1- \\
\text { Q3) }\end{array}$ & n' & Min-max & $\begin{array}{c}\text { Me (Q1- } \\
\text { Q3) }\end{array}$ & n' & Min-max & $\mathrm{Me}(\mathrm{Q} 1-\mathrm{Q} 3)$ & n' & $\operatorname{Min}-\max$ & K Me (Q1-Q3) & n' \\
\hline \multirow{2}{*}{$\begin{array}{l}\text { NGAL } \\
\text { (ng/mL) }\end{array}$} & Serum & $\begin{array}{l}25.00- \\
95.20\end{array}$ & $\begin{array}{l}50.50 \\
(38.80- \\
58.80)\end{array}$ & 0 & $\begin{array}{l}30.70- \\
201.90\end{array}$ & $\begin{array}{l}73.00 \\
(54.50- \\
129.70)\end{array}$ & 3 & $\begin{array}{l}27.20- \\
74.20\end{array}$ & $\begin{array}{l}45.80 \\
(33.70- \\
61.10) \\
\end{array}$ & 0 & $31.10-91.20$ & $\begin{array}{l}48.30 \\
(40.40- \\
57.30)\end{array}$ & 0 & $\begin{array}{l}27.20- \\
201.90\end{array}$ & $\begin{array}{l}51.10 \\
(40.40- \\
66.90) \\
\end{array}$ & 3 & $\begin{array}{l}50.60- \\
277.60\end{array}$ & $\begin{array}{l}98.10 \\
(59.40- \\
128.20) \\
\end{array}$ & 6 \\
\hline & Urine & $\begin{array}{l}8.50- \\
41.70\end{array}$ & $\begin{array}{l}20.70 \\
(17.90- \\
24.50) \\
\end{array}$ & 4 & $\begin{array}{l}12.90- \\
112.10\end{array}$ & $\begin{array}{l}53.40 \\
(31.10- \\
64.20) \\
\end{array}$ & 7 & $\begin{array}{l}18.80- \\
59.80\end{array}$ & $\begin{array}{l}30.20 \\
(23.10- \\
47.80) \\
\end{array}$ & 3 & $12.80-60.90$ & $\begin{array}{l}29.80 \\
(25.20- \\
33.00) \\
\end{array}$ & 2 & $\begin{array}{l}12.80- \\
112.10\end{array}$ & $\begin{array}{l}32.30 \\
(23.70- \\
55.80) \\
\end{array}$ & 12 & $\begin{array}{l}19.10- \\
223.00\end{array}$ & $\begin{array}{l}36.60 \\
(26.80- \\
89.90) \\
\end{array}$ & 7 \\
\hline \multirow{2}{*}{$\begin{array}{l}\text { Cystatin C } \\
(\mathrm{mg} / \mathrm{L})\end{array}$} & Serum & $0.13-0.71$ & $\begin{array}{l}0.25 \\
(0.19- \\
0.37)\end{array}$ & 2 & $0.09-1.01$ & $\begin{array}{l}0.36 \\
(0.19- \\
0.60) \\
\end{array}$ & 3 & $\begin{array}{r}0 \\
0.08-0.40 \\
0\end{array}$ & $\begin{array}{l}0.24 \\
(0.15- \\
0.26)\end{array}$ & 0 & $0.06-0.55$ & $\begin{array}{l}0.19 \\
(0.13- \\
0.26)\end{array}$ & 1 & $0.06-1.01$ & $\begin{array}{l}0.23(0.15- \\
0.37)\end{array}$ & 4 & $0.07-1.71$ & $\begin{array}{l}0.61(0.37- \\
1.13)\end{array}$ & 7 \\
\hline & Urine & $0.06-0.50$ & $\begin{array}{l}0.10 \\
(0.07- \\
0.13)\end{array}$ & 2 & $0.02-0.43$ & $\begin{array}{l}0.25 \\
(0.10- \\
0.35) \\
\end{array}$ & 5 & $\begin{array}{r}0 \\
0.07-0.19 \\
0\end{array}$ & $\begin{array}{l}0.13 \\
(0.12- \\
0.13)\end{array}$ & 0 & $0.05-0.34$ & $\begin{array}{l}0.14 \\
(0.08- \\
0.19)\end{array}$ & 1 & $0.02-0.43$ & $\begin{array}{l}0.13(0.10- \\
0.19)\end{array}$ & 6 & $0.10-0.50$ & $\begin{array}{l}0.34(0.22- \\
0.37)\end{array}$ & 9 \\
\hline
\end{tabular}

Me - median; Q1 - first quartile; Q3 - third quartile; S - serum; U - urine; n' - number of horses with elevated parameter above cut-off value

Table 2. Significant differences in serum and urine biomarkers between healthy horses (non-acute kidney injury (AKI) group), horses at risk of kidney injury (AKI-risk group; divided into colic sufferers, recipients of nonsteroidal anti-inflammatory drugs (NSAIDs) and recipients of gentamicin) and horses diagnosed with acute kidney injury (AKI group)

\begin{tabular}{|c|c|c|c|c|c|c|c|c|}
\hline \multirow{2}{*}{ Parameter } & & \multirow{2}{*}{$\begin{array}{c}\text { Non-AKI } v s \\
\text { AKI }\end{array}$} & \multicolumn{2}{|c|}{ AKI-risk colic $v s$} & \multicolumn{2}{|c|}{ AKI-risk NSAIDs $v s$} & \multicolumn{2}{|c|}{ AKI-risk gentamicin $v s$} \\
\hline & & & Non-AKI & AKI & Non-AKI & AKI & Non-AKI & AKI \\
\hline \multirow{2}{*}{$\begin{array}{l}\text { NGAL } \\
(\mathrm{ng} / \mathrm{mL})\end{array}$} & Serum & 0.0030 & - & - & - & 0.0030 & - & 0.0200 \\
\hline & Urine & 0.0050 & 0.0050 & - & - & - & - & - \\
\hline \multirow{2}{*}{$\begin{array}{l}\text { Cystatin C } \\
(\mathrm{mg} / \mathrm{L})\end{array}$} & Serum & 0.0400 & - & - & - & 0.0400 & - & 0.0200 \\
\hline & Urine & 0.0001 & 0.0400 & - & - & 0.0400 & - & 0.0300 \\
\hline
\end{tabular}

NGAL - neutrophil gelatinase-associated lipocalin

Table 3. Spearman's correlation between the analysed biomarkers and selected conventional renal dysfunction biomarkers

\begin{tabular}{|c|c|c|c|c|c|}
\hline & \multirow{2}{*}{ Parameter } & \multicolumn{2}{|l|}{ NGAL } & \multicolumn{2}{|l|}{ Cystatin C } \\
\hline & & Serum & Urine & Serum & Urine \\
\hline \multirow{2}{*}{ Serum } & Urea & $\begin{array}{l}r_{s}=0.360 \\
P=0.002\end{array}$ & NS & $\begin{array}{l}r_{s}=0.300 \\
P=0.014\end{array}$ & $\begin{array}{l}r_{s}=0.340 \\
P=0.004\end{array}$ \\
\hline & Creatinine & NS & NS & NS & $\begin{array}{l}\mathrm{r}_{\mathrm{s}}=0.300 \\
\mathrm{P}=0.013\end{array}$ \\
\hline \multirow{3}{*}{ Urine } & Protein & NS & $\begin{array}{l}r_{s}=0.360 \\
P<0.005\end{array}$ & NS & $\begin{array}{l}\mathrm{r}_{\mathrm{s}}=0.400 \\
\mathrm{P}<0.001\end{array}$ \\
\hline & GGT: Creatinine ratio & $\begin{array}{l}\mathrm{r}_{\mathrm{s}}=0.300 \\
\mathrm{P}=0.012\end{array}$ & $\begin{array}{l}\mathrm{r}_{\mathrm{s}}=0.390 \\
\mathrm{P}<0.001\end{array}$ & NS & $\begin{array}{l}\mathrm{r}_{\mathrm{s}}=0.370 \\
\mathrm{P}<0.005\end{array}$ \\
\hline & FENa & NS & $\begin{array}{l}\mathrm{r}_{\mathrm{s}}=0.33 \\
\mathrm{P}=0.005\end{array}$ & NS & NS \\
\hline
\end{tabular}

NGAL - neutrophil gelatinase-associated lipocalin; $r_{\mathrm{s}}$ - Spearman's correlation coefficient; GGT - Gamma-glutamyltransferase; FENa - Fractional sodium excretion; NS - statistically nonsignificant

Table 4. The receiver operating characteristic (ROC) analysis of serum and urinary cystatin $\mathrm{C}$ and neutrophil gelatinase-associated lipocalin (NGAL) relative to kidney dysfunction in horses

\begin{tabular}{llcccccc}
\hline Parameter & & Cut-off value & Sensitivity & Specificity & Positive predictive value & Negative predictive value & AUC \\
\hline NGAL & Serum & 95.20 & 0.54 & 0.93 & 0.60 & 0.92 \\
\hline & Urine & 33.10 & 0.64 & 0.71 & 0.30 & 0.90 \\
\hline Cystatin C & Serum & 0.53 & 0.64 & 0.85 & 0.44 & 0.66 \\
\hline & Urine & 0.20 & 0.82 & 0.85 & 0.50 & 0.93 \\
\hline
\end{tabular}

$\mathrm{AUC}$ - area under the ROC

\section{Discussion}

The conducted study presents the results of serum and urine cystatin $\mathrm{C}$ and serum and urine NGAL measured in healthy horses, horses at risk of AKI, and horses with AKI. To the authors' knowledge, this is the first study assessing NGAL and cystatin $\mathrm{C}$ in preclinical and clinical AKI in horses. The authors also assessed how selected biomarker concentrations corresponded to the concentrations of the more conventional biomarkers used as a standard indicator of renal function. The few current reports on new renal biomarkers in equine medicine focus mainly on serum biomarkers, while the present study assessed selected biomarkers in equine urine.

Neutrophil gelatinase-associated lipocalin is a small extracellular protein molecule the expression of which is rapidly induced in the nephron in response to renal epithelial hypoxia, injury and inflammation (13). Increased NGAL concentrations are seen in human patients with suspicion of AKI $(14,24)$. In horses, NGAL was identified in postmortem specimens of laminitic hoof lamellar tissue, necrotic cecum, inflamed salivary glands and wounds $(3,10)$. So far, there are only 
two reports on serum NGAL concentration in healthy horses and horses with renal problems $(9,27)$. Compared to the serum NGAL concentration in healthy horses reported by Jacobsen et al. (9), our concentration results were higher, but the maximums were similar (95.16 ng/mL vs $103.6 \mu \mathrm{g} / \mathrm{L})$, indicating similar upper limits of the norm. The serum NGAL concentration in the AKI-risk group was similar to the non-AKI group. The exception was the colic subgroup, where three horses had serum NGAL concentration above the cut-off value. This result may suggest the detection of early AKI. The highest concentrations of serum NGAL were observed in the AKI group. The median serum NGAL concentration in AKI horses was similar to that in horses with renal problems in the study by Jacobsen et al. (9). However, none of the AKI horses' results were as high as those reported previously. Instead, both the present investigation and the referenced study link the presence of a low concentration of NGAL and concomitant azotaemia. This can possibly be explained by different mechanisms leading to AKI in different cases (dysfunction or injury). Since the expression of NGAL occurs not only in renal tubules but also in other organs, nonrenal factors such as comorbid disease can have an impact on its concentration (29). Research by van Galen et al. (27) showed a higher concentration of serum NGAL in horses with renal problems and coexisting inflammation than in horses with renal problems alone. This makes serum NGAL a less sensitive marker for the detection of kidney disease in horses. Also, according to the results of the ROC analysis, serum NGAL proved to be more effective in identifying healthy individuals without the disease.

Increased urinary NGAL, in contrast to serum NGAL, indicates inadequate renal reabsorption ability (21). Since there are no published reference concentrations for urinary NGAL in horses, the results of this study were compared to those in the human medical literature. The urinary NGAL median concentration in non-AKI horses was similar to that recorded for humans, but the maximum values are lower (20). Interestingly, a few healthy horses had a urinary NGAL concentration above the cut-off value. In the AKI-risk group, the NGAL concentration was lower than those observed in people at risk of AKI (20). However, in colic horses urinary NGAL concentration was higher than in healthy horses, but the maximum concentration in this subgroup did not reach the values of the AKI group. This result can be compared to those in humans, where lower urinary NGAL values are observed in individuals at risk than in patients with clinical AKI (20). It can be concluded that urinary NGAL concentration increases with the transition of the renal disease from subclinical to clinical. Similarly to the other groups, urinary NGAL concentrations in AKI horses were lower than those reported in humans and their maximum concentrations did not reach human sufferer levels (20). Only seven AKI horses showed urinary NGAL concentration above the cut-off value. In humans, not all patients with clinical AKI had elevated NGAL urinary concentration, patients with stage 3 AKI having lower NGAL concentrations than those with stages 1 and 2 (20). This implies that urinary NGAL is elevated during early kidney disease and decreases with disease progression. This may also explain the lack of correlation between serum creatinine/urea and NGAL. In the examined equine subjects, there were horses with urinary NGAL above and serum NGAL below the cut-off value. It is possible that the concentration of NGAL increases in urine before it does in serum. Urinary NGAL showed greater sensitivity than the serum NGAL and the values obtained in the ROC analysis of this parameter are very similar to those obtained in humans (20).

Cystatin $\mathrm{C}$ is a protease inhibitor that is expressed in all nucleated cells and is excreted exclusively by the kidneys. Its concentrations are closely related to renal function (7) and AKI is predicted by elevated plasma cystatin C levels in critically ill patients (8). Only one study has researched cystatin $\mathrm{C}$ in horses (1). Our research documented serum cystatin $\mathrm{C}$ concentrations in the non-AKI group similar to those recorded in dogs and cats $(2,16,17)$. In the group of AKI-risk horses, serum cystatin $\mathrm{C}$ concentrations were no different from those in non-AKI horses. There were individuals with serum cystatin $\mathrm{C}$ concentration above the cut-off value. The highest concentration of serum cystatin $\mathrm{C}$ was achieved in the AKI horse group; however, while being the highest in the study, the reported concentrations in AKI horses were lower than those determined for other species with clinical renal disease, except cats (17). As in the case of NGAL, only some horses from this group showed values of this parameter above the cut-off value. Similar observations were made by Braun et al. (2) in dogs with signs of renal disease without azotaemia and in dogs without signs but with azotaemia. These results may be related to the severity of the disease, since in dogs, serum cystatin $\mathrm{C}$ levels increased with disease progression (16). However, due to no correlation having been seen between serum cystatin $C$ and serum creatinine in the study, this cannot be confirmed in horses. Cystatin $\mathrm{C}$ is also influenced by factors other than glomerular filtration rate, such as inflammation, metabolic diseases, and even the interval between feeding and serum collection $(2,4,25)$. Lack of significant correlations between serum cystatin $\mathrm{C}$ and other conventional renal dysfunction biomarkers may render this test only moderately powerful diagnostically.

Cystatin $\mathrm{C}$ is present in very negligible amounts in normal urine (15) and urinary concentrations of this protein for all examined horses in previous research were similar to the normal human range (15). In the AKI-risk group, five colic horses had urinary cystatin C concentrations above the cut-off value and in this subgroup this parameter was higher than in healthy horses. This may indicate the detection of early AKI in this group. In the AKI group of horses, as in the case of serum NGAL and cystatin $\mathrm{C}$, urinary cystatin $\mathrm{C}$ 
concentration was also the highest. Nine horses from this group had urine cystatin $\mathrm{C}$ values above the cut-off value. That elevated values were not common to all horses may be due to the different advancement of the renal dysfunction process in different individuals, as observed in humans (15). In our study, a correlation between cystatin $\mathrm{C}$ and serum creatinine was observed. Of all the tested biomarkers, urinary cystatin $\mathrm{C}$ had the highest sensitivity and diagnostic power.

Potential nephrotoxic side effects from colic and administration of nephrotoxic drugs are well documented in horses $(1,11,26)$. In the AKI-risk group, an increase in tested biomarkers was observed mainly in colic horses. An increase in several biomarkers in this group may indicate the occurrence of kidney dysfunction or organ injury. Since gastrointestinal diseases are associated with a higher number of changes in the animal - haemodynamic changes, endotoxemia and systematic inflammatory response syndrome - they have a potentially more significant negative impact on renal function (28). In horses receiving potentially nephrotoxic drugs, no changes in the novel biomarker were seen, compared to healthy horses. Complications associated with the use of nephrotoxic medication have been observed mainly in foals, young horses and horses with dehydration or endotoxaemia (5).

One of the study limitations was that the animals selected for the study were not homogeneous with respect to AKI aetiology or pathogenesis. This could influence the results of biomarker assays. However, under clinical conditions, AKI is polyaetiological in many patients (12). AKI itself may be associated with independent kidney injury with or without dysfunction. Moreover, a variety of biomarkers assess organ function and the degree of tissue damage. An optimally reliable predictive score for AKI may include a panel of different biomarkers rather than a single indicator. However, more research into the expression of selected biomarkers both in healthy and diseased horses is needed before they can be routinely used in practice. The second limitation of the study was the lack of renal histology, which would have allowed an assessment of the real degree of kidney injury in the examined horses. Due to the lack of available alternatives, serum creatinine was used as the gold standard to evaluate kidney function. Unfortunately, serum creatinine concentration is not an indicator capable of completely ruling out early AKI. The next limitation of the study was that biomarker concentrations were assessed only once. Each biomarker has its own concentration versus time curve. Finally, the storage of biological material, freezing or centrifugation of the sample may have affected the levels of the selected biomarkers. Due to the lack of other possibilities, the authors decided to use commercially available validated tests. The next interesting step would be to perform NGAL analyses with two different tests: those used by the authors and the ones used in the previous publications referred to. More research is needed to validate cystatin $\mathrm{C}$ and NGAL levels as biomarkers in horses at different stages of kidney disease progression.

In conclusion, in this study, horses with AKI had a higher median concentration of all biomarkers compared to healthy horses. None of the single selected biomarkers recognised azotaemia in all affected horses. In every study group there were individuals with values below and above the cut-off value, however the urinary NGAL and cystatin $\mathrm{C}$ concentrations were higher in AKI-risk horses with colic compared to healthy ones, which indicates that those parameters may be suitable for the detection of subclinical AKI. Gastrointestinal diseases had a greater impact on the changes in selected biomarkers, and thus in the kidneys. Since primary diseases can affect these biomarker concentrations, their use for recognising AKI may be limited. Urinary cystatin $\mathrm{C}$ showed the greatest diagnostic power as well as high sensitivity and specificity, which recommends further research be undertaken on this biomarker in horses. The obtained results indicate the need to create a serum and urinary biomarker panel to detect AKI instead of using a single selected biomarker.

Conflict of Interests Statement: The authors declare that there is no conflict of interests regarding the publication of this article.

Financial Disclosure Statement: This study was conducted as part of an "Innovative PHD" Ph.D. research programme (no. D220/0002/18) financially supported by the Wroclaw University of Environmental and Life Sciences.

Animal Rights Statement: The research on the horses with or at risk of AKI was in the purview of the Experiments on Animals Act of 15 January 2015 in paragraph 5 providing for "veterinary procedures which do not cause pain, suffering, distress or permanent health impairment more invasive than the insertion of a needle." and this obviated the need for Ethics Committee approval. Procedures on healthy horses were performed with the approval of the 2nd Local Ethics Committee on Animal Experimentation in Wrocław (permission no. 86/2018). The owners of all animals used in the research gave informed consent prior to the diagnostic procedures and publication of the results.

Acknowledgements: The authors acknowledge the help of Dr. Malwina Slowikowska, Prof. Artur Niedzwiedz, Krzysztof Kmiecik, Lukasz Oktawiec, Pawel Golonka, and Anastazja Lipski in assembling the studied groups.

\section{References}

1. Arosalo B.M., Raekallio M., Rajamäki M., Holopainen E., Kastevaara T., Salonen H., Sankari S.: Detecting early kidney damage in horses with colic by measuring matrix metalloproteinase -9 and -2 , other enzymes, urinary glucose and 
total proteins. Acta Vet Scand 2007, 49, 4, doi: 10.1186/17510147-49-4.

2. Braun J.P., Perxachs A., Péchereau D., de la Farge F.: Plasma cystatin $\mathrm{C}$ in the dog: Reference values and variations with renal failure. Comp Clin Pathol 2002, 11, 44-49, doi: 10.1007/s580002-8081-2.

3. Bundgaard L., Jacobsen S., Sørensen M.A., Sun Z., Deutsch E.W., Moritz R.L., Bendixen E.: The equine PeptideAtlas: a resource for developing proteomics-based veterinary research. Proteomics 2014, 4, 763-773, doi: 10.1002/pmic.201300398.

4. Cimerman N., Brguljan P.M., Krašovec M., Suskovic S., Kos J.: Twenty-four hour variations of plasma cystatin $\mathrm{C}$ and total cysteine proteinase inhibitory activity in sera from healthy patients. Clin Chim Acta 2000, 291, 89-95, doi:10.1016/S00098981(99)00196-5.

5. Gronwall R., Brown M.P., Hobbs S.: Serum gentamicin concentrations and pharmacokinetics in 2-week-old pony foals after intramuscular administration. J Equine Vet Sci. 1988, 8, 205-207, doi: 10.1016/S0737-0806(88)80003-0.

6. Groover E.S., Woolums A.R., Cole D.J., LeRoy B.E.: Risk factors associated with renal insufficiency in horses with primary gastrointestinal disease: 26 cases (2000-2003). J Am Vet Med Assoc 2006, 228, 572-577, doi: 10.2460/javma.228.4.572.

7. Halacova M., Kotaska K., Kukacka J., Vavrova V., Kuzelova M., Ticha J., Prusa R.: Serum cystatin C level for better assessment of glomerular filtration rate in cystic fibrosis patients treated by amikacin. J Clin Pharm Ther 2008, 33, 409-417, doi: 10.1111/j.1365-2710.2008.00932.x

8. Herget-Rosenthal S., Poppen D., Hüsing J., Marggraf G., Pietruck F., Jakob H.G., Philipp T., Kribben A.: Prognostic value of tubular proteinuria and enzymuria in nonoliguric acute tubular necrosis. Clin Chem 2004, 50, 552-558, doi: 10.1373/clinchem. 2003.027763.

9. Jacobsen S., Berg L.C., Tvermose E., Laurberg M.B., van Galen G.: Validation of an ELISA for detection of neutrophil gelatinaseassociated lipocalin (NGAL) in equine serum. Vet Clin Pathol 2018, 47, 603-607, doi: 10.1111/vcp.12670.

10. Laskoski L.M., Valadão C., Vasconcelos R.O., Faleiros R.R., Mendes H.M.F., Ferrucci D., Silva J.A.F., Machado D.D.R.S.: Neutrophil gelatinase associated lipocalin (NGAL) and calprotectin in equine laminar tissue after jejunal obstruction, treated or not with hydrocortisone (in Portuguese). Pesqui Vet Bras 2012, 32, 817-823, doi: 10.1590/S0100736X2012000900001.

11. MacAllister C.G., Morgan S.J., Borne A.T., Pollet R.A.: Comparison of adverse effects of phenylbutazone, flunixin meglumine, and ketoprofen in horses. J Am Vet Med Assoc 1993, 202, 71-77.

12. Makris K., Spanou L.: Acute Kidney Injury: definition, pathophysiology and clinical phenotypes. Clin Biochem Rev 2016, 37, 85-98.

13. Marchewka Z.: Low molecular weight biomarkers in the nephrotoxicity. Adv Clin Exp Med 2006, 15, 1129-1138.

14. Mishra J., Dent C., Tarabishi R., Mitsnefes M.M., Ma Q., Kelly C., Ruff S.M., Zahedi K., Shao M., Bean J., Mori K., Barasch J., Devarajan P.: Neutrophil gelatinase-associated lipocalin (NGAL) as a biomarker for acute renal injury after cardiac surgery. Lancet 2005, 365, 1231-1238, doi: 10.1016/S0140-6736(05)74811-X.

15. Park M.Y., Choi S.J., Kim J.K., Hwang S.D., Lee Y.W.: Urinary cystatin $\mathrm{C}$ levels as a diagnostic and prognostic biomarker in patients with acute kidney injury. Nephrology 2013, 18, 256-262, doi: 10.1111/nep.12037.

16. Pelander L., Häggström J., Larsson A., Syme H., Elliott J., Heiene R. Ljungvall I.: Comparison of the diagnostic value of symmetric dimethylarginine, cystatin $\mathrm{C}$, and creatinine for detection of decreased glomerular filtration rate in dogs. J Vet Intern Med 2019, 33, 630-639, doi: 10.1111/jvim.15445.

17. Poświatowska-Kaszczyszyn I.: Usefulness of serum cystatin C measurement for assessing renal function in cats. Bull Vet Inst Pulawy 2012, 56, 235-239, doi: 10.2478/v10213-012-0042-0.

18. R Core Team: R: A language and environment for statistical computing. R Foundation for Statistical Computing, Vienna, 2019. http://www.R-project.org.

19. Savage V.L., Marr C.M., Bailey M., Smith S.: Prevalence of acute kidney injury in a population of hospitalized horses. J Vet Intern Med 2019, 33, 2294-2301, doi: 10.1111/jvim/15569.

20. Schinstock C.A., Semret M.H., Wagner S.J., Borland T.M., Bryant S.C., Kashani K.B., Larson T.S., Lieske J.C.: Urinalysis is more specific and urinary neutrophil gelatinase-associated lipocalin is more sensitive for early detection of acute kidney injury. Nephrol Dial Transplant 2013, 28, 1175-1185, doi: $10.1093 / \mathrm{ndt} / \mathrm{gfs} 127$.

21. Schmidt-Ott K.M., Mori K., Li J.Y., Kalandadze A., Cohen D.J., Devarajan P., Barasch J.: Dual action of neutrophil gelatinaseassociated lipocalin. J Am Soc Nephrol 2007, 18, 407-413, doi: 10.1681/ASN.2006080882.

22. Schott H.C., Esser M.M.: The sick adult horse: renal clinical pathologic testing and urinalysis. Vet Clin North Am Equine Pract 2020, 36, 121-134, doi: 10.1016/j.cveq.2019.12.003.

23. Siwińska N., Pasławska U., Bąchor R., Szczepankiewicz B., Żak A., Grocholska P., Szewczuk Z.: Evaluation of podocin in urine in horses using qualitative and quantitative methods. PLoS One 2020, 15, e0240586, doi: 10.1371/journal.pone.0240586.

24. Srisawat N., Murugan R., Lee M., Kong L., Carter M., Angus D.C., Kellum J.A.: Plasma neutrophil gelatinase-associated lipocalin predicts recovery from acute kidney injury following communityacquired pneumonia. Kidney Int 2011, 80, 545-552, doi: 10.1038/ki.2011.160

25. Stevens L.A., Schmid C.H., Greene T., Li L., Beck G.J., Joffe M.M., Froissart M., Kusek J.W., Zhang Y., Coresh J., Levey A.S.: Factors other than glomerular filtration rate affect serum cystatin C levels. Kidney Int 2009, 75, 652-660, doi: 10.1038/ki.2008.638.

26. van der Harst M.R., Bull S., Laffont C.M., Klein W.R.: Gentamicin nephrotoxicity - a comparison of in vitro findings with in vivo experiments in equines. Vet Res Commun 2005, 29, 247-261, doi: 10.1023/B:VERC.0000047492.05882.bb.

27. van Galen G., Jacobsen S.V.A.M., Breinholt Laurberg M., Tvermose E., Broe-Brøndum R., Prior Theisen L., Berg L.C.: NGAL: a new biomarker in the horse for renal injury and inflammation. J Vet Intern Med 2019, 33, 1547-1560, doi: 10.1111/jvim. 15447 .

28. Wing D.A., Yamada T., Hawley H.B., Pettit G.W.: Model for disseminated intravascular coagulation: bacterial sepsis in rhesus monkeys. J Lab Clin Med 1978, 92, 239-251.

29. Xu S.Y., Pauksen K., Venge P.: Serum measurements of human neutrophil lipocalin (HNL) discriminate between acute bacterial and viral infections. Scand J Clin Lab Invest 1995, 55, 125-131, doi: 10.3109/00365519509089604.

30. Zhang W.R., Parikh C.R.: Biomarkers of acute and chronic kidney disease. Annu Rev Physiol 2019, 81, 309-333, doi: 10.1146/annurev-physiol-020518-114605. 